\title{
Equivalent diffusion coefficient and equivalent diffusion accessible porosity of a stratified porous medium
}

\author{
Marijke Huysmans • Alain Dassargues
}

Received: 4 August 2005 / Accepted: 16 March 2006

(C) Springer Science+Business Media B.V. 2007

\begin{abstract}
Diffusion is an important transport process in low permeability media, which play an important role in contamination and remediation of natural environments. The calculation of equivalent diffusion parameters has however not been extensively explored. In this paper, expressions of the equivalent diffusion coefficient and the equivalent diffusion accessible porosity normal to the layering in a layered porous medium are derived based on analytical solutions of the diffusion equation. The expressions show that the equivalent diffusion coefficient changes with time. It is equal to the power average with $p=-0.5$ for small times and converges to the harmonic average for large times. The equivalent diffusion accessible porosity is the harmonic average of the porosities of the individual layers for all times. The expressions are verified numerically for several test cases.
\end{abstract}

Keywords Diffusion - Equivalent parameters · Low permeability media

\section{List of symbols}

$C\left(\mathrm{~kg} / \mathrm{m}^{3}\right) \quad$ solute concentration in the liquid phase.

$D\left(\mathrm{~m}^{2} / \mathrm{s}\right) \quad$ effective diffusion coefficient (as defined by Gillham et al. 1984).

$D_{\text {eq }}\left(\mathrm{m}^{2} / \mathrm{s}\right)$ equivalent diffusion coefficient.

$L(\mathrm{~m}) \quad$ layer thickness.

$n(-) \quad$ diffusion accessible porosity.

$n_{\text {eq }}(-) \quad$ equivalent diffusion accessible porosity.

$R(-) \quad$ retardation factor.

M. Huysmans $(\bowtie) \cdot$ A. Dassargues

Hydrogeology and Engineering Geology Group, Department of Geology-Geography, Katholieke Universiteit Leuven, Redingenstraat 16, 3000 Leuven, Belgium

e-mail: marijke.huysmans@geo.kuleuven.ac.be

\section{A. Dassargues}

Hydrogeology and Environmental Geology, Department of Georesources, Geotechnologies and Building Materials (GEOMAC)., Université de Liège, B 52/3, 4000 Liège, Belgium

e-mail: alain.dassargues@geo.kuleuven.ac.be 
$t(\mathrm{~s})$ time.

$x(\mathrm{~m})$ distance from the top of the first layer.

$\bar{X}_{p} \quad$ power average with power $=p$.

\section{Introduction}

Transport by diffusion is defined as transport due to a concentration gradient of solute. This transport mechanism is usually not modeled in aquifers since it is a much slower process than transport by advection and dispersion. In media with a low hydraulic conductivity, however, advection velocities may be very small, so that diffusion may be the dominant transport mechanism under typically encountered conditions of hydraulic gradient (Desaulniers et al. 1981; Johnson et al. 1989; Shackelford and Daniel 1991). Such low permeability media have traditionally received less attention in hydrogeological practice and research. Such media may however play an important role in the contamination and remediation of natural environments. Aquitard contamination can for example result in a tailing or rebound effect on the remediation of an aquifer overlying the aquitard since contamination transport in aquitards is a very slow process (Liu and Ball 1998). Low permeability media are also important as host formations for disposal of municipal (e.g., Jang and Kim 2003), industrial (e.g., Navarro et al. 2000) and nuclear waste (e.g., Landais 2004).

As low permeability media receive increasing interest, extensive databases of diffusion coefficients and diffusion accessible porosities are collected (e.g., Aertsens et al. 2004; Descostes et al. 2004; Patriarche et al. 2004; Van Loon et al. 2003). Figures 1 and 2 show examples of measured diffusion coefficients and diffusion accessible porosities versus depth from two clay layers which are studied in the context of nuclear waste disposal. As information about the spatial variability and heterogeneity of diffusion parameters becomes available, questions arise about the appropriate average value of the diffusion coefficient and the diffusion accessible porosity, representative for a given volume of the medium. The aim is to assign a single effective or equivalent value of the diffusion parameters to the medium, so that the total solute flux is equal to the flux in the heterogeneous medium. This approach of equivalent diffusion parameters is for example interesting when diffusion fluxes through a heterogeneous medium should be calculated if the model grid scale is larger than the scale of heterogeneity due to computation time issues. Equivalent diffusion parameters are also very useful when the retention capacity of several potential waste disposal sites is compared in preliminary site selection without sophisticated numerical models of the different heterogeneous media. Insight in equivalent diffusion parameters is also very helpful for the interpretation of diffusion tests in heterogeneous media.

A lot of research has been carried out about equivalent flow and transport parameters. The majority of this research focuses on equivalent values for hydraulic conductivity. Renard and de Marsily (1997) presented a review of the many studies about the equivalent permeability for steady-state, uniform and single-phase flow. Several authors also investigated effective hydraulic conductivity for transient radial flow (Barker and Herbert 1982; Butler 1988; Butler and McElwee 1990; Oliver 1990; Indelman 2003). Relatively few studies have been carried out about other equivalent flow and transport parameters than hydraulic conductivity. Gelhar and Axness (1983), Dagan $(1982,1984,1987,1988)$ and Neuman et al. (1987) employed different analytical approaches to derive expressions for macrodispersivity coefficients which depend on

Springer 


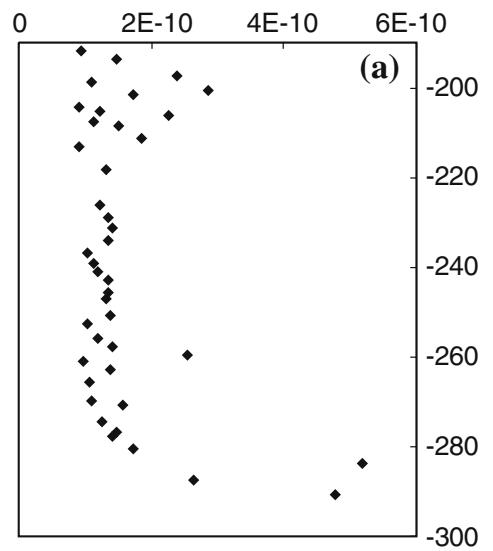

Diffusion coefficient iodide $D\left(\mathrm{~m}^{2} / \mathrm{s}\right)$

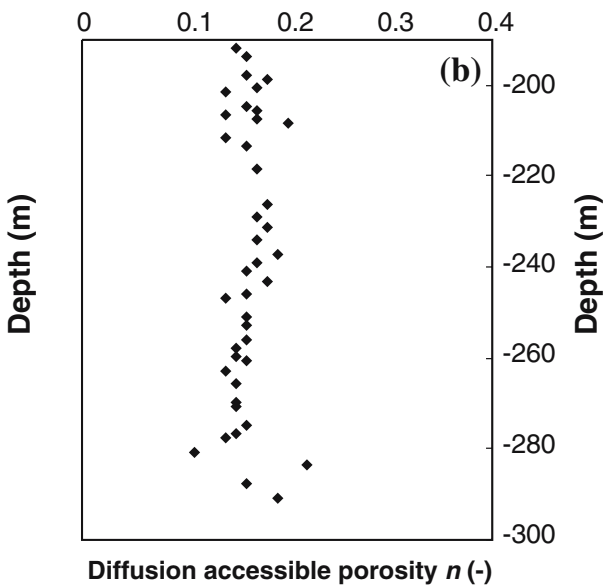

Fig. 1 (a) Diffusion coefficient $D\left[\mathrm{~m}^{2} / \mathrm{s}\right]$ and (b) diffusion accessible porosity $n[-]$ of iodide in the Boom Clay, Belgium (Aertsens et al. 2004)
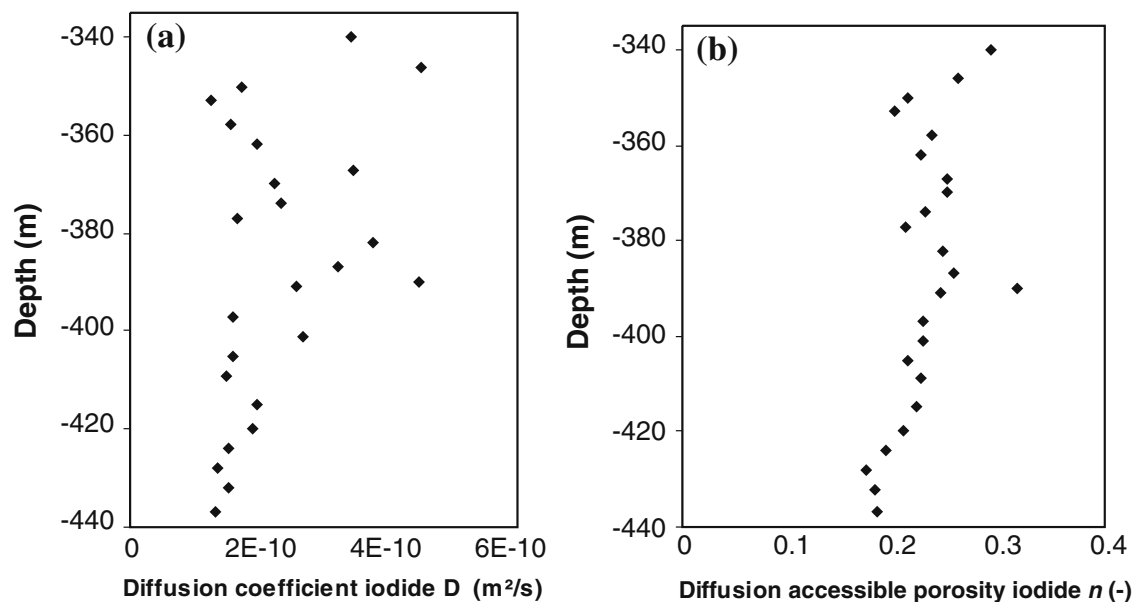

Fig. 2 (a) Diffusion coefficient $D\left[\mathrm{~m}^{2} / \mathrm{s}\right]$ and (b) diffusion accessible porosity $n[-]$ of iodide in the Ieper Clay, Belgium (Van Marcke and Laenen 2005)

the mean flow gradient and the statistics of the log hydraulic conductivity field. Didierjean et al. (2004) studied the effective porosity and the effective dispersion coefficient of a periodic stratified porous medium. Several authors studied effective sorption parameters (Roberts et al. 1986; Cvetkovic and Shapiro 1990; Kabala and Sposito 1991; Chrysikopoulos et al. 1992; Hu et al. 1995; Ptak and Schmid 1996; Metzger et al. 1996; Reichle et al. 1998). The discussion of the effective diffusion coefficient has been limited to the steady-state case (Özisik 1993), in which the same expressions hold as for the mathematically similar processes of steady-state groundwater or heat flow. The study of diffusion is however particularly interesting in the transient state, since the spreading of a contaminant plume is inherently a transient phenomenon.

This paper investigates the calculation of the equivalent diffusion coefficient and the equivalent diffusion accessible porosity of a layered porous medium in function of 
time. Equivalent properties of layered porous media are especially interesting since several important low permeability layers show a distinct layering caused by their geological deposition conditions, e.g., Boom Clay, Belgium (Aertsens et al. 2004), Kimmeridge clay formation, UK (Chambers et al. 2000) and Opalinus Clay, Switzerland (Van Loon et al. 2003). Section 2 describes the problem concept and the governing equations. Section 3 recalls the expressions of the equivalent diffusion coefficient and the equivalent diffusion accessible porosity in steady-state. Section 4 describes the derivation of expressions for equivalent diffusion parameters from the transient solution of the diffusion equations. In Section 5, the expressions are verified with numerical calculations. Section 6 summarizes the main conclusions of this study.

\section{Problem formulation}

The problem concept is based on the common situations of a landfill located on top of a low permeability layer (Fig. 3a) or an underground nuclear waste disposal site located in the middle of a low permeability formation (Fig. 3b). The size of the landfill or the underground waste disposal site is assumed to be aerially large compared to the thickness of the low permeability layer, so that only one-dimensional vertical diffusion should be considered. The low permeability layer is a stratified medium composed of a finite number of sublayers. Initially, a medium consisting of only two layers is considered, with layer 1 on top and layer 2 on the bottom. Layers 1 and 2 are assumed to be individually homogeneous with respect to their diffusion parameters, but each with its own constant effective diffusion coefficient $D_{1}$ and $D_{2}\left(\mathrm{~m}^{2} / \mathrm{s}\right)$, its own constant retardation factor $R_{1}$ and $R_{2}(-)$ and its own constant diffusion accessible porosity $n_{1}$ and $n_{2}(-)$.

The $1 \mathrm{D}$ vertical diffusion equations in the two layers are:

$$
\begin{gathered}
R_{1} \frac{\partial C_{1}}{\partial t}=D_{1} \frac{\partial^{2} C_{1}}{\partial x^{2}} \quad 0<x<L_{1} \quad t>0 \\
R_{2} \frac{\partial C_{2}}{\partial t}=D_{2} \frac{\partial^{2} C_{2}}{\partial x^{2}} \quad L_{1}<x<L_{2} \quad t>0
\end{gathered}
$$

(a)

(b)
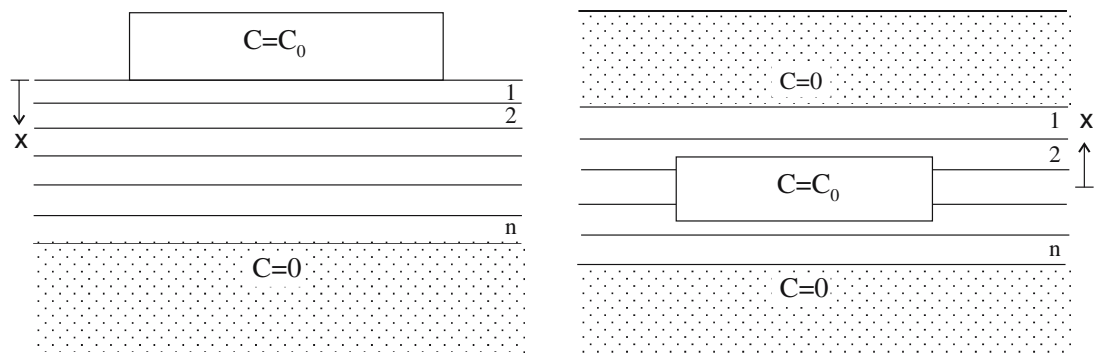

Fig. 3 Problem concept of (a) a landfill located on top of a layered low permeability medium and (b) an underground nuclear waste disposal site located in the middle of a layered low permeability formation 
where $C_{1}$ and $C_{2}$ are the solute concentration in the liquid phase $\left(\mathrm{kg} / \mathrm{m}^{3}\right)$ in the first and second layer respectively, $t$ is time (s), $x$ is distance from the top of the first layer (m) and $L_{1}$ and $L_{2}$ are the thicknesses of the layers $(\mathrm{m})$. In the remainder of the manuscript, it is assumed that no retardation occurs, i.e., $R_{1}=R_{2}=1$.

The boundary concentration at the top of the two-layer medium (scenario of Fig. $3 a$ ) or at the source inside the medium (scenario of Fig. 3b) is prescribed and assumed to be a known constant concentration $C_{0}\left(\mathrm{~kg} / \mathrm{m}^{3}\right)$.

$$
C_{1}(x=0, t)=C_{0}
$$

This boundary condition is e.g. applied in high-level nuclear waste disposal in low permeability media with radionuclide sources that remain for a large time period and that can be modeled as constant concentration sources with a specified concentration equal to the solubility limit (e.g., Mallants et al. 2001).

At the interface between the two layers, continuity of concentration and mass flux are imposed.

$$
\begin{gathered}
C_{1}\left(x=L_{1}, t\right)=C_{2}\left(x=L_{1}, t\right) \\
\left.n_{1} D_{1} \frac{\partial C_{1}}{\partial x}\right|_{x=L_{1}}=\left.n_{2} D_{2} \frac{\partial C_{2}}{\partial x}\right|_{x=L_{1}}
\end{gathered}
$$

The boundary concentration at the bottom of the two-layer medium is prescribed and assumed to be zero. This is a common boundary condition on aquitard-aquifer interfaces in diffusion dominated problems, since it is assumed that solutes reaching the aquifer are immediately flushed away by advection.

$$
C_{2}\left(x=L_{1}+L_{2}\right)=0
$$

The initial concentrations of the contaminant in layers 1 and 2 are assumed to be zero.

$$
\begin{aligned}
& C_{1}(x, t=0)=0 \\
& C_{2}(x, t=0)=0
\end{aligned}
$$

The equivalence condition is that the flux leaving at the bottom of the equivalent medium should be equal to the flux leaving at the bottom of the two-layer medium. The equivalent diffusion coefficient and the equivalent diffusion accessible porosity are defined by imposing that the flux at $x=L_{1}+L_{2}$ in the equivalent medium with an equivalent diffusion coefficient $D_{\text {eq }}\left(\mathrm{m}^{2} / \mathrm{s}\right)$ and an equivalent diffusion accessible porosity $n_{\mathrm{eq}}(-)$ should be equal to the flux at $x=L_{1}+L_{2}$ in the two-layer medium.

$$
\begin{gathered}
F_{\text {eq }}\left(L_{1}+L_{2}\right)=F_{1,2}\left(L_{1}+L_{2}\right) \\
-\left.n_{\mathrm{eq}} D_{\mathrm{eq}} \frac{\partial C(x, t)}{\partial x}\right|_{x=L_{1}+L_{2}}=-\left.n_{2} D_{2} \frac{\partial C_{2}(x, t)}{\partial x}\right|_{x=L_{1}+L_{2}}
\end{gathered}
$$

where $C$ is the solute concentration in the liquid phase $\left(\mathrm{kg} / \mathrm{m}^{3}\right)$ in the one-layer equivalent medium. Equation (10) shows that the equivalent diffusion coefficient and the equivalent diffusion accessible porosity can be dependent on time. 


\section{Steady state solution}

In this section, the steady state solution of the problem is discussed. This solution is reached in the asymptotic limit. In steady state, concentration varies linearly in each layer (Ozisik 1993). The two-layer solution is given by:

$$
\begin{array}{ll}
C_{1}=C_{0}-\frac{n_{2} D_{2} C_{0}}{n_{1} D_{1} L_{2}+n_{2} D_{2} L_{1}} x & 0 \leq x \leq L_{1} \\
C_{2}=\frac{n_{1} D_{1} C_{0}}{n_{1} D_{1} L_{2}+n_{2} D_{2} L_{1}}\left(L_{1}+L_{2}-x\right) & L_{1} \leq x \leq L_{2}
\end{array}
$$

The spatial concentration distribution in the equivalent medium is given by:

$$
C=C_{0}\left(1-\frac{x}{\left(L_{1}+L_{2}\right)}\right) 0 \leq x \leq\left(L_{1}+L_{2}\right)
$$

The equivalent diffusion coefficient and diffusion accessible porosity in steady state, can be found by imposing that the fluxes at $x=L_{1}+L_{2}$ must be equal:

$$
\begin{aligned}
n_{2} D_{2} \frac{\partial C_{2}}{\partial x} & =n_{\text {eq }} D_{\text {eq }} \frac{\partial C}{\partial x} \Leftrightarrow n_{2} D_{2} \frac{n_{1} D_{1} C_{0}}{n_{1} D_{1} L_{2}+n_{2} D_{2} L_{1}}=n_{\text {eq }} D_{\text {eq }} \frac{C_{0}}{L_{1}+L_{2}} \\
& \Leftrightarrow n_{\text {eq }} D_{\text {eq }}=\frac{n_{1} D_{1} n_{2} D_{2}\left(L_{1}+L_{2}\right)}{n_{1} D_{1} L_{2}+n_{2} D_{2} L_{1}}
\end{aligned}
$$

This shows that $n_{\mathrm{eq}} D_{\text {eq }}$ is the weighted harmonic mean of $n_{1} D_{1}$ and $n_{2} D_{2}$, as previously reported by Ash et al. (1963), Barrie et al. (1963) and Barrer (1968). This result is identical to the equation for effective hydraulic conductivity in layered media with flow perpendicular to the stratification. This is logical since Fick's law describing diffusion is analogous to Darcy's law. This result also holds for stratified media with more than two layers, since the derivations can easily be extended. Equation (13) also shows that there is no unique solution for $n_{\mathrm{eq}}$ and $D_{\text {eq }}$ since this is a single equation with two unknowns. For every value of $n_{\text {eq }}, D_{\text {eq }}$ can be determined so that $n_{\text {eq }} D_{\text {eq }}$ is the weighted harmonic mean of $n_{1} D_{1}$ and $n_{2} D_{2}$, and vice versa.

\section{Transient solution}

There is no general simple analytical solution to the formulated two-layer transient problem. There is however such a solution available for a slightly different problem, in which layer 2 is a semi-infinite layer with a contaminant flux assumed to be zero at infinite depth.

$$
\left.\frac{\partial C_{2}}{\partial x}\right|_{x=+\infty}=0
$$

For short times, the solution to this semi-infinite problem is equal to the solution to the earlier formulated finite problem. This is true since, for short times, the contaminant plume is very small compared to the layer thickness. In that case, it makes no difference whether the boundary condition is applied at a finite or infinite distance. This is illustrated in Fig. 4 that shows a comparison between the analytical solution to the semi-infinite problem (Luikov 1968; Liu and Ball 1998) and the numerical 


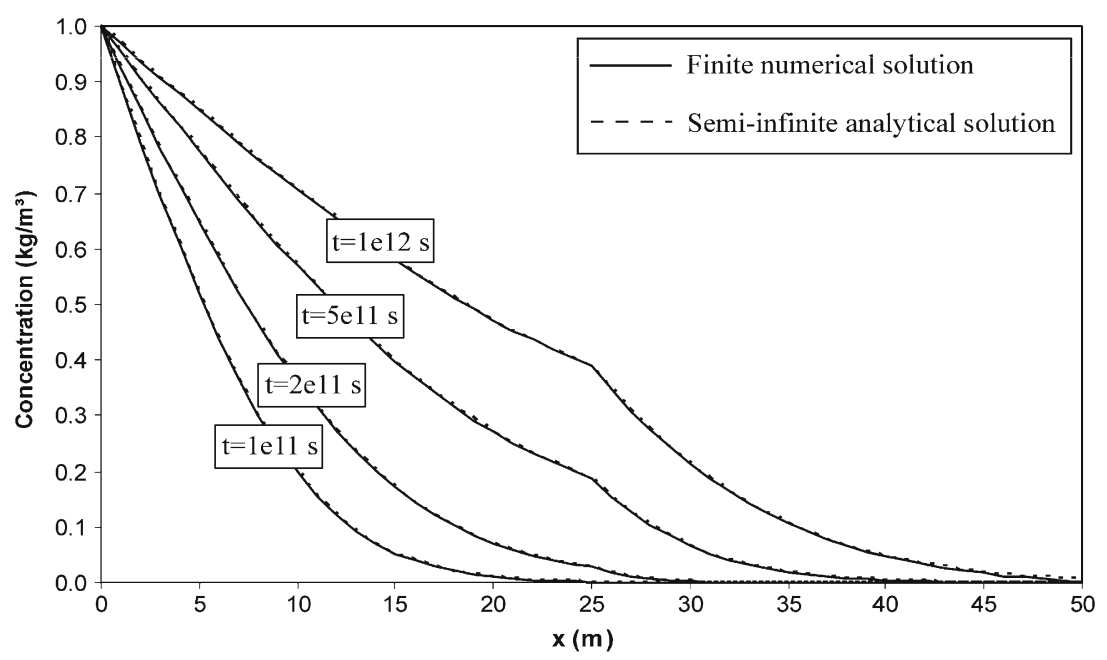

Fig. 4 Comparison of the finite numerical solution and the semi-infinite analytical solution for diffusion in a two-layer medium with $n=0.3, D_{1}=3 \mathrm{e}-10 \mathrm{~m}^{2} / \mathrm{s}, D_{2}=1 \mathrm{e}-10 \mathrm{~m}^{2} / \mathrm{s}, L_{1}=L_{2}=25 \mathrm{~m}$

solution for the finite problem for an example with realistic parameter input values. No difference is observed between the two solutions for times smaller than $10^{12} \mathrm{~s}$ (i.e. more than 30,000 years). It can therefore be assumed that equivalent parameter expressions derived from the semi-infinite analytical solution also hold for the finite problem for short times.

The analytical solution to this semi-infinite problem with initial conditions (7) and (8) and boundary conditions (3) and (14) is given by (Luikov 1968; Liu and Ball 1998):

$$
\begin{gathered}
C_{1}(x, t)=C_{0} \sum_{k=0}^{+\infty} \theta^{k}\left[\operatorname{erfc}\left(\frac{\sqrt{R_{1}}\left(2 k L_{1}+x\right)}{2 \sqrt{D_{1} t}}\right)-\theta \operatorname{erfc}\left(\frac{\sqrt{R_{1}}\left((2 k+2) L_{1}-x\right)}{2 \sqrt{D_{1} t}}\right)\right] \\
C_{2}(x, t)=(1-\theta) C_{0}\left[\sum_{k=0}^{+\infty} \theta^{k} \operatorname{erfc}\left(\frac{\sqrt{R_{1} / D_{1}}(2 k+1) L_{1}+\sqrt{R_{2} / D_{2}}\left(x-L_{1}\right)}{2 \sqrt{t}}\right)\right] \\
\text { where } \theta=\frac{n_{2} \sqrt{D_{2} R_{2}}-n_{1} \sqrt{D_{1} R_{1}}}{n_{2} \sqrt{D_{2} R_{2}}+n_{1} \sqrt{D_{1} R_{1}}}
\end{gathered}
$$

If both layers have the same property $(\theta=0)$, then the solution becomes the wellknown one-layer solution:

$$
C(x, t)=C_{0} \sqrt{\frac{R x^{2}}{4 \pi D}} \int_{0}^{t} \frac{1}{\sqrt{(t-\tau)^{3}}} \exp \left(-\frac{R x^{2}}{4 D(t-\tau)}\right) d \tau
$$

For mathematical simplicity, we first investigate the equivalent diffusion coefficient assuming that the diffusion accessible porosities of both layers are equal and the 
equivalent diffusion accessible porosity assuming that the diffusion coefficients of both layers are equal. Secondly, we investigate if the expressions obtained with these simplified assumptions also hold in the case where both the diffusion coefficient and the diffusion accessible porosity are variable and possibly correlated.

Inserting Eq. (16) and the one-layer analytical solution in the definition of equivalent diffusion coefficient (10) assuming that the diffusion accessible porosities of both layers are equal, gives:

$$
\begin{aligned}
& \frac{n C_{0} \sqrt{D_{\mathrm{eq}}}}{\sqrt{\pi t}} \exp \left(-\frac{\left(L_{1}+L_{2}\right)^{2}}{4 D_{\mathrm{eq}} t}\right) \\
= & \frac{n \sqrt{D_{2}}(1-\theta)}{\sqrt{\pi t}} C_{0} \sum_{k=0}^{+\infty} \theta^{k} \exp \left[-\left(\frac{\sqrt{1 / D_{1}}(2 k+1) L_{1}+\sqrt{1 / D_{2}} L_{2}}{2 \sqrt{t}}\right)^{2}\right]
\end{aligned}
$$

The infinite sum in the right-hand term converges relatively fast (Liu and Ball 1998). The condition that must be satisfied for convergence with only one term is that the second term is much smaller in absolute value than the first term, or if

$$
\frac{2 L_{1}^{2}}{D_{1} t}+\frac{L_{1} L_{2}}{\sqrt{D_{1} D_{2}} t} \gg|\ln | \frac{\sqrt{D_{2}}-\sqrt{D_{1}}}{\sqrt{D_{2}}+\sqrt{D_{1}}}||
$$

For short times and if $D_{1}$ and $D_{2}$ are not too different, this condition is usually satisfied. For example, if $D_{2}$ is three times larger than $D_{1}\left(D_{1}=10^{-10} \mathrm{~m}^{2} / \mathrm{s}\right.$ and $D_{2}=3 \times 10^{-10} \mathrm{~m}^{2} / \mathrm{s}$ ) and if $L_{1}=L_{2}=25 \mathrm{~m}$, the left hand side of Eq. (20) is more than ten times larger than the right hand side of the equation for times smaller than 38,000 years. If condition (20) is satisfied, the expression for $D_{\text {eq }}$ becomes:

$$
\sqrt{D_{\text {eq }}} \exp \left(-\frac{\left(L_{1}+L_{2}\right)^{2}}{4 D_{\text {eq }} t}\right)=\frac{2 \sqrt{D_{1} D_{2}}}{\sqrt{D_{1}}+\sqrt{D_{2}}} \exp \left[-\left(\frac{L_{1} / \sqrt{D_{1}}+L_{2} / \sqrt{D_{2}}}{2 \sqrt{t}}\right)^{2}\right]
$$

If $L_{1}=L_{2}=L$, the expression for $D_{\text {eq }}$ becomes:

$$
\sqrt{D_{\text {eq }}} \exp \left(-\frac{L^{2}}{D_{\text {eq }} t}\right)=\frac{2 \sqrt{D_{1} D_{2}}}{\sqrt{D_{1}}+\sqrt{D_{2}}} \exp \left[-\left(\frac{L / \sqrt{D_{1}}+L / \sqrt{D_{2}}}{2 \sqrt{t}}\right)^{2}\right]
$$

The solution to this equation is:

$$
D_{\text {eq }}=\left(\frac{2 \sqrt{D_{1} D_{2}}}{\sqrt{D_{1}}+\sqrt{D_{2}}}\right)^{2}
$$

The equivalent diffusion coefficient of two layers with the same thickness for short times is thus the power average with $p=-0.5$ of the diffusion coefficients of the two layers for the considered set of boundary conditions. The power average of a set of $n$ sample measurements $X_{1}, X_{2}, \ldots, X_{n}$ is defined as (Jensen 1998):

$$
\begin{array}{ll}
\bar{X}_{p}=\left(\frac{1}{n} \sum_{i=1}^{n} X_{i}^{p}\right)^{1 / p} & p \neq 0 \\
\bar{X}_{p}=\exp \left\{\left[\sum \ln \left(X_{i}\right)\right] / n\right\} & p=0
\end{array}
$$


For the special cases where $p=-1,0$ or 1 , the power average is the harmonic, geometric or arithmetic mean respectively.

Inserting the analytical solution of the semi-infinite problem in the definition of equivalent diffusion accessible porosity (10) assuming that the diffusion coefficients of both layers are equal, gives:

$$
\begin{aligned}
& \frac{n_{\mathrm{eq}} C_{0} \sqrt{D}}{\sqrt{\pi t}} \exp \left(-\frac{\left(L_{1}+L_{2}\right)^{2}}{4 D t}\right) \\
& =\frac{2 n_{1} n_{2}}{n_{1}+n_{2}} \frac{\sqrt{D}}{\sqrt{\pi t}} C_{0} \sum_{k=0}^{+\infty}\left(\frac{n_{2}-n_{1}}{n_{2}+n_{1}}\right)^{k} \exp \left[-\left(\frac{(2 k+1) L_{1}+L_{2}}{2 \sqrt{D t}}\right)^{2}\right]
\end{aligned}
$$

The infinite sum in the right-hand term converges relatively fast (Liu and Ball 1998). One term is sufficient if the second term is much smaller in absolute value than the first term, or if

$$
\frac{2 L_{1}^{2}+L_{1} L_{2}}{D t} \geq|\ln | \frac{n_{2}-n_{1}}{n_{2}+n_{1}}||
$$

For small times and if $n_{1}$ and $n_{2}$ are not too different, this condition is usually satisfied. For example, if $n_{2}$ is four times larger than $n_{1}\left(n_{1}=0.1\right.$ and $\left.n_{2}=0.4\right)$, if $D=2 \times 10^{-10} \mathrm{~m}^{2} / \mathrm{s}$ and if $L_{1}=L_{2}=25 \mathrm{~m}$, the left hand side of Eq. (26) is more than 10 times larger than the right hand side of the equation for times smaller than 57,000 years. If condition (26) is satisfied, the expression for $n_{\mathrm{eq}}$ becomes:

$$
n_{\mathrm{eq}}=\frac{2 n_{1} n_{2}}{n_{1}+n_{2}}
$$

The equivalent diffusion accessible porosity is then the harmonic mean of $n_{1}$ and $n_{2}$.

Expressions (23) and (27) respectively give the equivalent diffusion coefficients if the diffusion accessible porosities of both layers are equal and the equivalent diffusion accessible porosity if the diffusion coefficients of both layers are equal. In the second part of this paragraph, we investigate the more realistic case where both the diffusion coefficient and the diffusion accessible porosity are variable and possibly correlated.

Inserting the analytical solution of the semi-infinite problem in the definition of the equivalent diffusion coefficient and the equivalent diffusion accessible porosity (10), gives:

$$
\begin{aligned}
& \frac{n_{\mathrm{eq}} C_{0} \sqrt{D_{\mathrm{eq}}}}{\sqrt{\pi t}} \exp \left(-\frac{\left(L_{1}+L_{2}\right)^{2}}{4 D_{\mathrm{eq}} t}\right) \\
& =-\frac{C_{0}}{\sqrt{\pi t}}\left(\frac{2 n_{1} n_{2} \sqrt{D_{1} D_{2}}}{n_{2} \sqrt{D_{2}}+n_{1} \sqrt{D_{1}}}\right) \sum_{k=0}^{+\infty}\left(\frac{n_{2} \sqrt{D_{2}}-n_{1} \sqrt{D_{1}}}{n_{2} \sqrt{D_{2}}+n_{1} \sqrt{D_{1}}}\right)^{k} \\
& \exp \left[-\left(\frac{\sqrt{1 / D_{1}}(2 k+1) L_{1}+\sqrt{1 / D_{2} L_{2}}}{2 \sqrt{t}}\right]\right.
\end{aligned}
$$

The infinite sum in the right-hand term converges relatively fast (Liu and Ball 1998). One term is sufficient if the second term is much smaller in absolute value than the first term, or if 


$$
\frac{2 L_{1}^{2} / D_{1}+L_{1} L_{2} / \sqrt{D_{1} D_{2}}}{t} \gg|\ln | \frac{n_{2} \sqrt{D_{2}}-n_{1} \sqrt{D_{1}}}{n_{2} \sqrt{D_{2}}+n_{1} \sqrt{D_{1}}}||
$$

For short times, if $D_{1}$ and $D_{2}$ are not too different and if $n_{1}$ and $n_{2}$ are not too different, this condition is usually satisfied. For example, if $D_{2}$ is three times larger than $D_{1}\left(D_{1}=10^{-10} \mathrm{~m}^{2} / \mathrm{s}\right.$ and $\left.D_{2}=3 \times 10^{-10} \mathrm{~m}^{2} / \mathrm{s}\right)$, if $n_{2}$ is four times larger than $n_{1}$ ( $n_{1}=0.1$ and $n_{2}=0.4$ ) and if $L_{1}=L_{2}=25 \mathrm{~m}$, the left hand side of Eq. (29) is more than 10 times larger than the right hand side of the equation for times smaller than 175,000 years. If Eq. (29) is satisfied, the expression for $D_{\text {eq }}$ and $n_{\text {eq }}$ becomes:

$$
\begin{aligned}
& n_{\text {eq }} \sqrt{D_{\text {eq }}} \exp \left(-\frac{\left(L_{1}+L_{2}\right)^{2}}{4 D_{\text {eq }} t}\right) \\
& =\frac{2 n_{1} n_{2} \sqrt{D_{1} D_{2}}}{n_{2} \sqrt{D_{2}}+n_{1} \sqrt{D_{1}}} \exp \left[-\left(\frac{\sqrt{1 / D_{1}} L_{1}+\sqrt{1 / D_{2}} L_{2}}{2 \sqrt{t}}\right)^{2}\right]
\end{aligned}
$$

If $L_{1}=L_{2}=L$, the expression for $D_{\text {eq }}$ and $n_{\text {eq }}$ becomes:

$$
n_{\mathrm{eq}} \sqrt{D_{\text {eq }}} \exp \left(-\frac{L^{2}}{D_{\text {eq }} t}\right)=\frac{2 n_{1} n_{2} \sqrt{D_{1} D_{2}}}{n_{2} \sqrt{D_{2}}+n_{1} \sqrt{D_{1}}} \exp \left[-\left(\frac{\sqrt{1 / D_{1}} L+\sqrt{1 / D_{2}} L}{2 \sqrt{t}}\right)^{2}\right]
$$

This equation has no unique solution for $n_{\mathrm{eq}}$ and $D_{\mathrm{eq}}$ since this is a single equation with two unknowns. For every value of $n_{\text {eq }}, D_{\text {eq }}$ can be determined so that Eq. (31) is satisfied, and vice versa. Moreover, the solutions for $n_{\mathrm{eq}}$ and $D_{\text {eq }}$ to this equation can generally not be expressed analytically. One particular solution to this equation is especially interesting since it can be expressed analytically. If $D_{\text {eq }}$ is chosen to be equal to the power average with $p=-0.5$ of the diffusion coefficients of the two layers, solution of Eq. (31) gives the following expression for $n_{\mathrm{eq}}$ :

$$
n_{\mathrm{eq}}=\frac{n_{1} n_{2}\left(\sqrt{D_{1}}+\sqrt{D_{2}}\right)}{n_{1} \sqrt{D_{1}}+n_{2} \sqrt{D_{2}}}=\left(\frac{\sqrt{D_{2}} /\left(\sqrt{D_{1}}+\sqrt{D_{2}}\right)}{n_{1}}+\frac{\sqrt{D_{1}} /\left(\sqrt{D_{1}}+\sqrt{D_{2}}\right)}{n_{2}}\right)^{-1}
$$

so that $n_{\mathrm{eq}}$ is equal to the harmonic mean of the diffusion accessible porosities of the two layers weighted with the square root of the diffusion coefficient of the other layer divided by the sum of the square roots of the diffusion coefficients of the two layers.

\section{Numerical verification}

In this section, these analytical results are verified with a numerical model. Initially, the results for the equivalent diffusion coefficient and the diffusion accessible porosity are tested in cases where either the diffusion coefficient or the diffusion accessible porosity is constant. Secondly, the analytical solutions are also numerically verified for the more realistic case where both the diffusion coefficient and the diffusion accessible porosity are variable and possibly correlated. The analytical solutions are in both cases verified in a two-layer medium with equal layer thicknesses and in stratified media with more than two layers or with layers with unequal thicknesses. All calculated examples have a realistic variability of $D$ and $n$ that corresponds to the 
natural variability of diffusion parameters observed in low permeability media (e.g., Shackelford and Daniel 1991; Aertsens et al. 2004; Patriarche et al. 2004).

The numerical model is a model of $50 \mathrm{~m} \times 1 \mathrm{~m} \times 1 \mathrm{~m}$ with cell dimensions of $1 \mathrm{~m} \times 1 \mathrm{~m} \times 1 \mathrm{~m}$. A constant arbitrary concentration equal to 1 is prescribed at $x=0$ and a constant zero concentration is prescribed at $x=50 \mathrm{~m}$. The other model boundaries are no flux boundaries. The total runtime is $5 \times 10^{13} \mathrm{~s}$ and the time step size is $1 \times 10^{10} \mathrm{~s}$. The effect of time step and grid cell size was examined and it was shown that smaller time steps and cell dimensions do not lead to different results. This model is run with FRAC3DVS, a finite element simulator for three-dimensional groundwater flow and solute transport in porous, discretely fractured porous or dual-porosity formations (Therrien et al. 1996, Therrien et al. 2003). For different cases, equivalent diffusion coefficients and diffusion accessible porosities are calculated by inverse modeling at different times. The equivalent diffusion coefficient and diffusion accessible porosity of a one-layer medium are iteratively adapted until the output flux of the equivalent one-layer medium differs at most $0.1 \%$ from the output flux of the considered multi-layer medium.

Initially, the results for the equivalent diffusion coefficient are tested for a two-layer medium with equal layer thicknesses $L_{1}=L_{2}=25 \mathrm{~m}$. The diffusion accessible porosity is set to 0.3 and the diffusion coefficients of the two layers are $3 \times 10^{-10} \mathrm{~m}^{2} / \mathrm{s}$ and $1 \times 10^{-10} \mathrm{~m}^{2} / \mathrm{s}$ respectively. The theoretical analysis of the previous sections has shown that in that case $D_{\text {eq }}$ goes from the power mean with $p=-0.5$ for small times to the harmonic mean as $t$ increases. The equivalent diffusion coefficient of this two-layer medium is calculated by inverse modeling at different times. The equivalent diffusion coefficient was calculated more frequently for small times since it displays more rapid change in time for these small times (Fig. 5). It is confirmed from these results that the calculated equivalent diffusion coefficient is the power mean with $p=-0.5$ for small times, and decreases to the harmonic mean as $t$ increases, as predicted by the analytical solutions.

The results for the equivalent diffusion accessible porosity are also tested in a twolayer medium with equal layer thicknesses $L_{1}=L_{2}=25 \mathrm{~m}$. The diffusion coefficient is set to $1.6 \times 10^{-10} \mathrm{~m}^{2} / \mathrm{s}$ and the diffusion accessible porosities of the two layers are 0.4 and 0.1 respectively. The theoretical analysis of the previous sections has shown that in that case the equivalent diffusion accessible porosity $n_{\text {eq }}$ is equal to the harmonic mean for small and large times. The equivalent diffusion accessible porosity of this two-layer medium is calculated by inverse modeling at different times. This calculation confirmed that the calculated equivalent diffusion accessible porosity is the harmonic mean for all times, as predicted by the analytical solutions.

Next, it is checked whether the equations for the equivalent diffusion coefficient also apply for stratified media with more than two layers. Transport by diffusion is modeled through a 10-layer medium. Each layer has a different diffusion coefficient between $5 \times 10^{-11} \mathrm{~m}^{2} / \mathrm{s}$ and $3 \times 10^{-10} \mathrm{~m}^{2} / \mathrm{s}$. The diffusion accessible porosity is set to 0.3 . The equivalent diffusion coefficient of this 10 -layer medium is calculated by inverse modeling at different times (Fig. 6). It can be observed that $D_{\text {eq }}$ decreases similarly in function of time as in the case of a two-layer medium. For large times, $D_{\text {eq }}$ converges to the harmonic average of the diffusion coefficients of the 10 layers. For small times, $D_{\text {eq }}$ approaches a value slightly larger than the power mean with $p=-0.5$. The largest $p$-value is approximately equal to -0.47 . This slightly larger equivalent diffusion coefficient can be explained by the dependence of transient diffusion on the order of the layers (Barrie et al. 1963). For very small times, the diffusion coefficient 

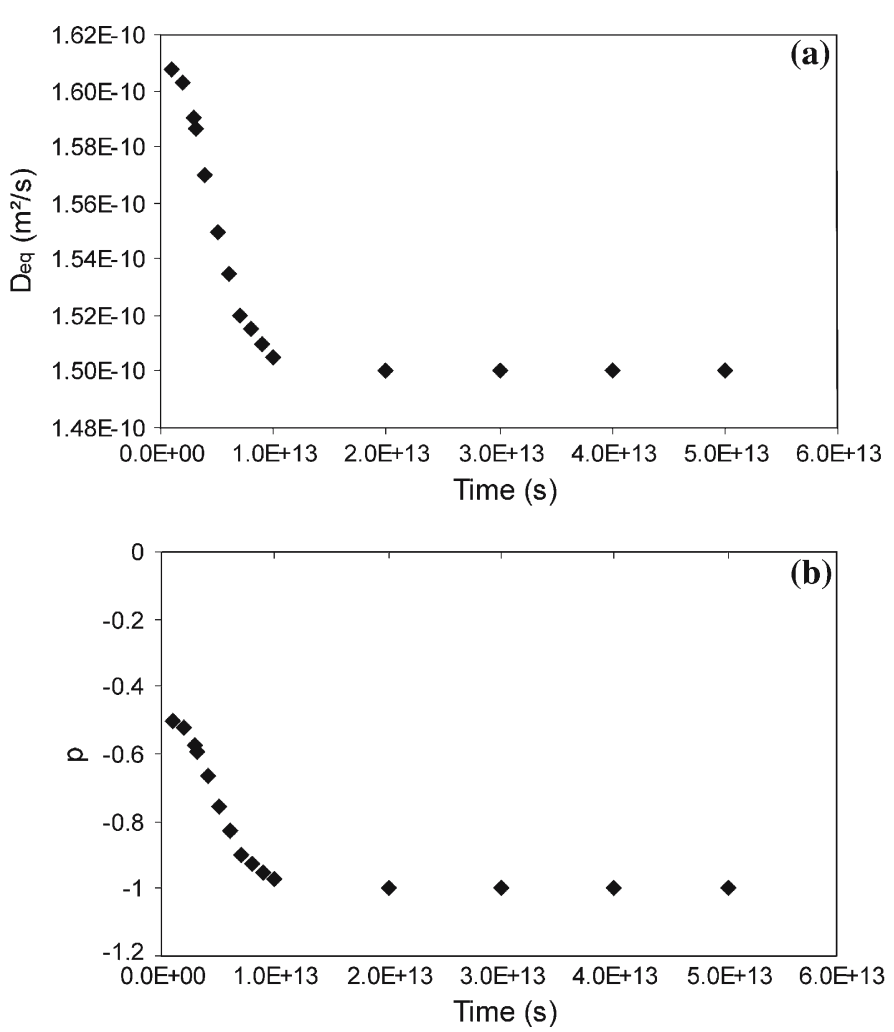

Fig. 5 Equivalent diffusion coefficient $D_{\text {eq }}(\mathbf{a})$ and power average exponent $p(\mathbf{b})$ in function of time for a two-layer medium with $n=0.3, D_{1}=3 \mathrm{e}-10 \mathrm{~m}^{2} / \mathrm{s}, D_{2}=1 \mathrm{e}-10 \mathrm{~m}^{2} / \mathrm{s}, L_{1}=L_{2}=25 \mathrm{~m}$

of the first layer plays a relatively larger role compared to diffusion coefficients of the other layers. In this example, the first layer has the largest diffusion coefficient of all 10 layers and therefore the equivalent diffusion coefficient for very small times is slightly larger than the power mean with $p=-0.5$. This effect is however very small, partially caused by the limited, but realistic, variability of the diffusion coefficient.

The results for the equivalent diffusion accessible porosity are also tested in a 10-layer medium. Each layer has a different diffusion accessible porosity between 0.25 and 0.35 . The diffusion coefficient is set to $1.6 \times 10^{-10} \mathrm{~m}^{2} / \mathrm{s}$. The equivalent diffusion accessible porosity of this 10-layer medium is calculated by inverse modeling at different times. The calculated equivalent diffusion accessible porosity is close to the harmonic average for all times. For very small times, the calculated equivalent diffusion accessible porosity is $1 \%$ larger than the harmonic average.

Next, it is checked whether the equations for the equivalent diffusion coefficient also apply for stratified media with layers of unequal thickness. The diffusion accessible porosity is set to 0.3 , layer 1 and 2 are 20 and $30 \mathrm{~m}$ thick respectively and the diffusion coefficients of the two layers are $3 \times 10^{-10} \mathrm{~m}^{2} / \mathrm{s}$ and $10^{-10} \mathrm{~m}^{2} / \mathrm{s}$ respectively. The equivalent diffusion coefficient of this two-layer medium is calculated by inverse modeling at different times (Fig. 7). As before, the calculated equivalent diffusion coefficient converges to the weighted harmonic average of the two diffusion coefficients. For small times, the calculated equivalent diffusion coefficient is about $7 \%$ 
larger than the weighted power average with $p=-0.5$. The power average exponent $p$ is close to -0.4 instead of -0.5 for small time. This shows that in stratified media with layers of unequal thickness, the equivalent diffusion coefficient is not exactly equal to the weighted harmonic average of the two diffusion coefficients but can be closely approximated by it.

The results for the equivalent diffusion accessible porosity are also tested in a two-layer medium with layers of unequal thickness. The diffusion coefficient is set to $1.6 \times 10^{-10} \mathrm{~m}^{2} / \mathrm{s}$, layer 1 and 2 are 20 and $30 \mathrm{~m}$ thick respectively and the diffusion accessible porosities of the two layers are 0.35 and 0.25 respectively. The equivalent diffusion accessible porosity of this two-layer medium is calculated by inverse modeling at different times. The equivalent diffusion accessible porosity is for all times very close to the harmonic average. For very small times, it is about $3 \%$ larger than the harmonic average.

The analytical solutions for the diffusion coefficient and the diffusion accessible porosity are also tested in media where both diffusion parameters are variable. The theoretical analysis of the previous sections has shown that in that case there is no unique solution for $n_{\mathrm{eq}}$ and $D_{\text {eq }}$ since the equivalence condition results in a single equation with two unknowns. Two particular solutions are verified numerically: the solution for small times where $D_{\text {eq }}$ is chosen to be equal to the power average with
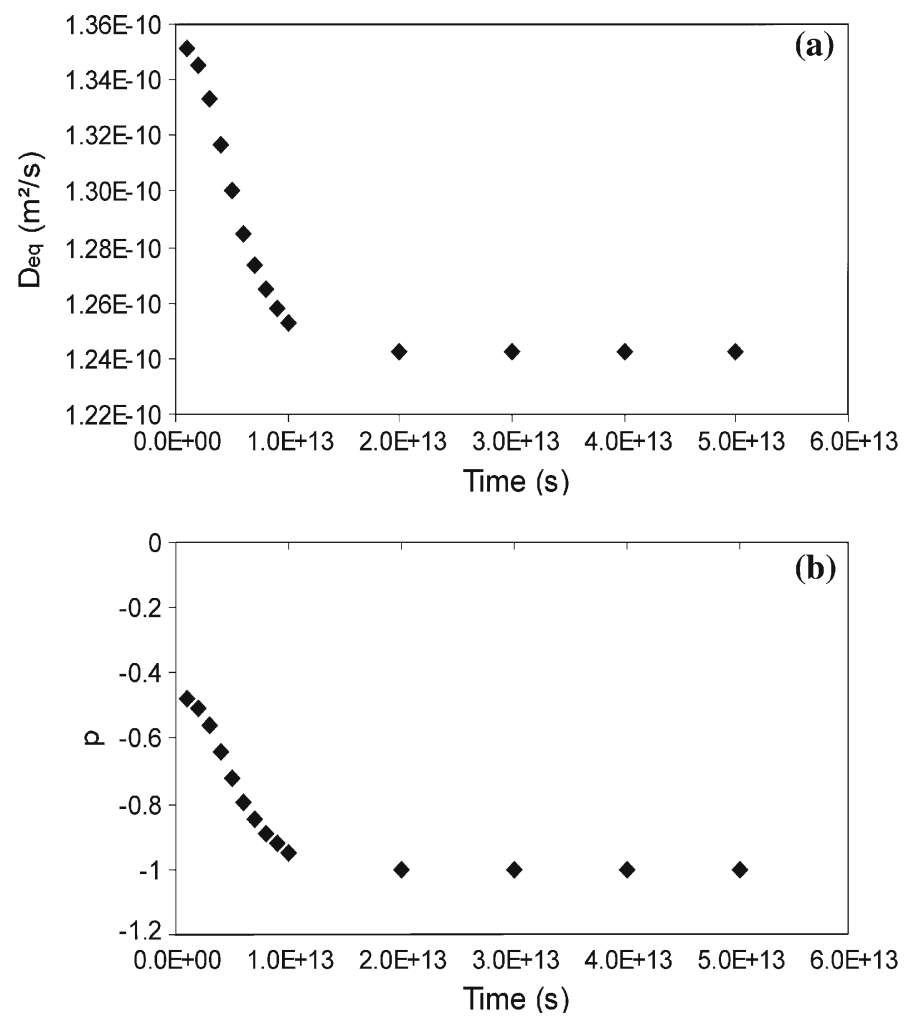

Fig. 6 Equivalent diffusion coefficient $D_{\text {eq }}(\mathbf{a})$ and power average exponent $p$ (b) in function of time for a 10-layer medium with $n=0.3, D_{i}$ between $5 \mathrm{e}-11$ and $3 \mathrm{e}-10 \mathrm{~m}^{2} / \mathrm{s}, L_{i}=5 \mathrm{~m}$ 
$p=-0.5$ of the diffusion coefficients of the two layers and $n_{\mathrm{eq}}$ is given by Eq. (32) and the solution for large times where $n_{\mathrm{eq}}$ is chosen to be equal to the harmonic mean of the porosities of the individual layers and $D_{\text {eq }}$ is calculated by Eq. (13).

The analytical solutions for the equivalent diffusion coefficient and the equivalent diffusion accessible porosity are first tested for a two-layer medium with equal layer thicknesses $L_{1}=L_{2}=25 \mathrm{~m}$, diffusion coefficients of $3 \times 10^{-10} \mathrm{~m}^{2} / \mathrm{s}$ and $1 \times 10^{-10} \mathrm{~m}^{2} / \mathrm{s}$ and diffusion accessible porosities of 0.4 and 0.1 . The flux leaving at the bottom of the two-layer medium and the equivalent medium are calculated numerically for the small time solution (Eq. (31)) and the large time solution (Eq. (13)). For small times $\left(t=1 \times 10^{12} \mathrm{~s}\right)$, there is a difference of $0.22 \%$ between the flux in the two-layer medium and the flux in the equivalent medium with equivalent properties given by the small time solution. For large times $\left(t>2 \times 10^{13} \mathrm{~s}\right)$, there is difference smaller than $0.02 \%$ between the flux in the two-layer medium and the flux in the equivalent medium with equivalent properties given by the large time solution. The analytical solutions are thus very good approximations for the equivalent diffusion coefficient and diffusion accessible porosity in a two-layer medium with equal layer thicknesses with variable $D$ and $n$.

The analytical solutions for the equivalent diffusion coefficient and the equivalent diffusion accessible porosity are also tested for a two-layer medium with unequal layer thicknesses $\left(L_{1}=20 \mathrm{~m}\right.$ and $\left.L_{2}=30 \mathrm{~m}\right)$, diffusion coefficients of $3 \times 10^{-10} \mathrm{~m}^{2} / \mathrm{s}$ and
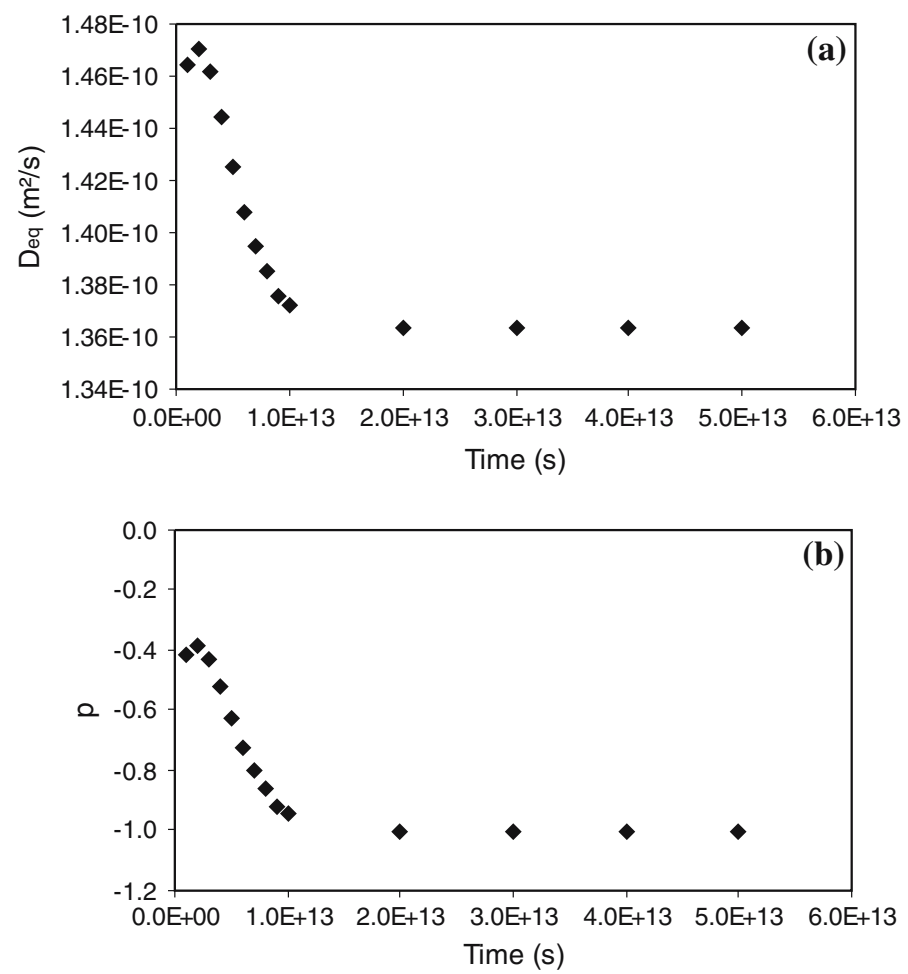

Fig. 7 Equivalent diffusion coefficient $D_{\text {eq }}(\mathbf{a})$ and power average exponent $p(\mathbf{b})$ in function of time for a two-layer medium with $n=0.3, D_{1}=3 \mathrm{e}-10 \mathrm{~m}^{2} / \mathrm{s}, D_{2}=1 \mathrm{e}-10 \mathrm{~m}^{2} / \mathrm{s}, L_{1}=20 \mathrm{~m}, L_{2}=30 \mathrm{~m}$ 
$1 \times 10^{-10} \mathrm{~m}^{2} / \mathrm{s}$ and diffusion accessible porosities of 0.35 and 0.25 . The flux leaving at the bottom of the two-layer medium and the equivalent medium are calculated numerically for the small time solution and the large time solution. For small times $\left(t=1 \times 10^{12} \mathrm{~s}\right)$, there is a difference of $0.07 \%$ between the flux in the two-layer medium and the flux in the equivalent medium with equivalent properties given by the small time solution. For large times $\left(t>2 \times 10^{13} \mathrm{~s}\right)$, there is difference smaller than $0.01 \%$ between the flux in the two-layer medium and the flux in the equivalent medium with equivalent properties given by the large time solution. The analytical solutions are thus also very good approximations for the equivalent diffusion coefficient and diffusion accessible porosity in a two-layer medium with unequal layer thicknesses with variable $D$ and $n$.

The analytical solutions for the equivalent diffusion coefficient and the equivalent diffusion accessible porosity are finally also tested for a ten-layer medium. Each layer has a different diffusion coefficient between $5 \times 10^{-11} \mathrm{~m}^{2} / \mathrm{s}$ and $3 \times 10^{-10} \mathrm{~m}^{2} / \mathrm{s}$ and a different diffusion accessible porosity between 0.25 and 0.35 . The flux leaving at the bottom of the 10-layer medium and the equivalent medium are calculated numerically for the small time solution and the large time solution. For small times $\left(t=1 \times 10^{12} \mathrm{~s}\right)$, there is a difference of $0.27 \%$ between the flux in the 10-layer medium and the flux in the equivalent medium with equivalent properties given by the small time solution. For large times $\left(t>2 \times 10^{13} \mathrm{~s}\right)$, there is difference smaller than $0.01 \%$ between the flux in the two-layer medium and the flux in the equivalent medium with equivalent properties given by the large time solution. The analytical solutions are thus also very good approximations for the equivalent diffusion coefficient and diffusion accessible porosity in a medium with more than ten layers and with variable $D$ and $n$.

These examples show that the analytical solutions are good approximations for the equivalent diffusion coefficient and diffusion accessible porosity under different conditions. The expressions are tested for media with two or more layers, with equal and unequal layer thicknesses and with one or two variable diffusion parameters. All calculated examples have a realistic variability of $D$ and $n$ that corresponds to the natural variability of diffusion parameters observed in low permeability media. The good agreement between the calculated fluxes in the heterogeneous media and the calculated fluxes in the equivalent homogeneous media shows that the natural variability of $D$ and $n$ is usually small enough to fulfill condition (29).

\section{Conclusions}

Expressions for the equivalent diffusion coefficient and the equivalent diffusion accessible porosity in a stratified medium have been derived. The expressions assume onedimensional diffusion normal to the layering and prescribed constant concentration boundary conditions at the top and bottom of the layered medium. Two media are considered equivalent if the diffusion fluxes leaving the bottom of the medium are equal. The expressions show that the equivalent diffusion coefficient changes with time. If diffusion accessible porosity is assumed constant, the equivalent diffusion coefficient is equal to the power average with $p=-0.5$ for short times and converges to the harmonic average for long times. If diffusion coefficient is assumed constant, the equivalent diffusion accessible porosity is the harmonic average of the porosities of the individual layers for all times. If both the diffusion coefficient and the porosity are 
variable, there is no unique solution for $n_{\mathrm{eq}}$ and $D_{\mathrm{eq}}$ since the equivalence condition results in a single equation with two unknowns. For small times, one particular solution is interesting since it can be expressed analytically, i.e., the solution where $D_{\text {eq }}$ is chosen to be equal to the power average with $p=-0.5$ of the diffusion coefficients of the two layers and $n_{\mathrm{eq}}$ is the harmonic mean of the diffusion accessible porosities of the two layers weighted with the square root of the diffusion coefficient of the other layer divided by the sum of the square roots of the diffusion coefficients of the two layers. For long times, $n_{\mathrm{eq}} D_{\mathrm{eq}}$ is the weighted harmonic mean of the products of $n$ and $D$ of the individual layers.

The expressions were verified numerically for several test cases in which the variability of $D$ and $n$ was not too large. The numerical results agreed well with analytically derived expressions. It should be noted that the values of the equivalent diffusion coefficient and the equivalent diffusion accessible porosity may be different for other boundary conditions. The effect of boundary conditions on these equivalent diffusion parameters is an interesting subject for further research.

Acknowledgements The authors wish to acknowledge the Fund for Scientific Research-Flanders for providing a Research Assistant scholarship to the first author.

\section{References}

Aertsens, M., Wemaere, I., Wouters, L.: Spatial variability of transport parameters in the Boom Clay. Appl. Clay Sci. 26, 37-45 (2004)

Ash, R., Barrer, R.M., Petropoulos, J.H.: Diffusion in heterogeneous media: properties of a laminated slab. Br. J. Appl. Phys. 14, 854-862 (1963)

Barker, J.A., Herbert, R.: Pumping tests in patchy aquifers. Ground Water 20, 130-155 (1982)

Barrer, R.M.: Diffusion and permeation in heterogeneous media (Chapter 6), in Crank, J., Park, G.S. (eds.) Diffusion in Polymers, pp. 165-215. Academic Press, New York (1968)

Barrie, J.A., Levind, J.D., Michaels, A.S., Wong, P.: Diffusion and solution of gases in composite rubber membranes. Trans. Faraday Soc. 59, 854-862 (1963)

Butler, J.J. Jr.: Pumping tests in nonuniform aquifers: the radially symmetric case. J. Hydrol. 101, 15-30 (1988)

Butler, J.J. Jr. McElwee, C.D.: Variable rate pumping tests for radially symmetric nonuniform aquifers. Water Resour. Res. 26, 291-306 (1990)

Chambers, M.H., Lawrence, D.S.L., Sellwood, B.W., Parker, A.: Annual layering in the Upper Jurassic Kimmeridge clay formation, UK, quantified using an ultra-high resolution SEM-EDX investigation. Sediment Geol 137, 9-23 (2000)

Chrysikopoulos, C.V., Kitanidis, P.K., Roberts, P.V.: Macrodispersion of sorbing solutes in heterogeneous porous formations with spatially periodic retardation factor and velocity field. Water Resour. Res. 28, 1517-1529 (1992)

Cvetkovic, V., Shapiro, A.: Mass arrival of sorptive solute in heterogeneous porous media. Water Resour. Res. 26, 2057-2067 (1990)

Dagan, G.: Stochastic modeling of groundwater flow by unconditional and conditional probabilities, 2, The solute transport. Water Resour. Res. 18, 835-848 (1982)

Dagan, G.: Solute transport in heterogeneous porous formations. J. Fluid Mech. 145, 151-177 (1984)

Dagan, G.: Theory of solute transport by groundwater. Annu. Rev. Fluid Mech. 19, 183-215 (1987)

Dagan, G.: Time-dependent macrodispersion for solute transport in anisotropic heterogeneous aquifers. Water Resour. Res. 24, 1491-1500 (1988)

Desaulniers, D.E., Cherry, J.A., Fritz, P.: Origin, age and movement of pore water in argillaceous quaternary deposits at four sites in southwestern Ontario. J. Hydrol. 50, 231-257 (1981)

Descostes, M., Blin, V., Grenut, B., Meier, P., Pointeau, I., Tevissen, E.: I-125(-) and Cl-36(-) diffusion in Oxfordian limestones and Callovo-Oxfordian argillites formations. Geochim Cosmochim Acta 68(11), A507-A507 (2004) 
Didierjean, S., Maillet, D., Moyne, C.: Analytical solutions of one-dimensional macrodispersion in stratified porous media by the quadrupole method: convergence to an equivalent homogeneous porous medium. Adv. Water Res. 27, 657-667 (2004)

Gelhar, L.W., Axness, C.L.: Three-dimensional stochastic analysis of macrodispersion in aquifers. Water Resour. Res. 19, 161-180 (1983)

Gillham, R.W., Robin, M.J.L., Dytynyshyn, D.J., Johnston, H.M.: Diffusion of nonreactive and reactive solutes through fine-grained barrier materials. Can. Geotech. J. 21(3), 541-550 (1984)

Hu, B.X., Deng, F.W., Cushman, J.H.: Nonlocal reactive transport with physical and chemical heterogeneity - linear nonequilibrium sorption with random Kd. Water Resour. Res. 31, 2239-2252 (1995)

Indelman, P.: Transient well-type flows in heterogeneous formations. Water Resour. Res. 39(3), 1064, doi:10.1029/2002WR001407 (2003)

Jang, Y.-S., Kim, Y.-I.: Behavior of a municipal landfill from field measurement data during a wastedisposal period. Env. Geol. 44, 592-598 (2003)

Jensen, J.L.: Some statistical properties of power averages for lognormal samples. Water Resour. Res. 34, 2415-2418 (1998)

Johnson, R.L., Cherry, J.A., Pankow, J.F.: Diffusive contaminant transport in natural clay: a field example and implications for clay-lined waste disposal sites. Environ. Sci. Technol. 23, 340-349 (1989)

Kabala, Z.J., Sposito, G.: A stochastic model of reactive solute transport with time-varying velocity in a heterogeneous aquifer. Water Resour. Res. 27, 341-350 (1991)

Landais, P.: Clays in natural and engineered barriers for radioactive waste confinement. Appl. Clay Sci. 26(1-4), 1 (2004)

Liu, C., Ball, W.P.: Analytical modeling of diffusion-limited contamination and decontamination in a two-layer porous medium. Adv. Water Resour. 21, 297-313 (1998)

Luikov, A.V.: Analytical Heat Diffusion Theory, 2nd edn. Academics Press, New York (1968)

Mallants, D., Marivoet, J., Sillen, X.: Performance assessment of the disposal of vitrified high-level waste in a clay layer. J. Nucl. Mat. 298, 125-135 (2001)

Metzger, D., Kinzelbach, H., Kinzelbach, W.: Effective dispersion of a solute cloud in a chemically heterogeneous porous medium: comparison of two ensemble-averaging procedures. Water Resour. Res. 32, 3311-3319 (1996)

Navarro, J.A.S., Lopez, C., Garcia, A.P.: Characterization of groundwater flow in the Bailin hazardous waste-disposal site (Huesca, Spain). Env. Geol. 40(1-2), 216-222 (2000)

Neuman, S.P., Winter, C.L., Newman, C.M.: Stochastic theory of field-scale Fickian dispersion in anisotropic porous media. Water Resour. Res. 23, 453-466 (1987)

Oliver, D.S.: The average process in permeability estimation from well-test data. SPE Form. Eval. 5, 319-324 (1990)

Özisik, M.N.: Heat Conduction, 2nd edn. Wiley, New York (1993)

Patriarche, D., Michelet, J.-L., Ledoux, E., Savoye, S.: Diffusion as the main process for mass transport in very low water content argillites: 1 . Chloride as a natural tracer for mass transport-Diffusion coefficient and concentration measurements in interstitial water. Water Resour. Res. 40(1), W01517, doi:10.1029/2003WR002700 (2004)

Ptak, T., Schmid, G.: Dual-tracer transport experiments in a physically and chemically heterogeneous porous aquifer: effective transport parameters and spatial variability. J. Hydrol. 183, 117-138 (1996)

Reichle, R., Kinzelbach, W., Kinzelbach, H.: Effective parameters in heterogeneous and homogeneous transport models with kinetic sorption. Water Resour. Res. 34, 583-594 (1998)

Renard, P., de Marsily, G.: Calculating equivalent permeability: a review. Adv. Water Resour. 20, 253-278 (1997)

Roberts, P.V., Goltz, M.N., Mackay, D.M.: A natural gradient experiment on solute transport in a sand aquifer, 3, Retardation estimates and mass balances for organic solutes. Water Resour. Res. 22, 2047-2058 (1986)

Shackelford, C.D., Daniel, D.E.: Diffusion in saturated soil, I, Background. J. Geotech. Eng. 117, 467-484 (1991)

Therrien, R., Sudicky, E.A.: Three-dimensional analysis of variably-saturated flow and solute transport in discretely-fractured porous media. J. Cont. Hydrol. 23 (1-2), 1-44 (1996)

Therrien, R., Sudicky, E.A., McLaren, R.G.: FRAC3DVS: An Efficient Simulator for Three-dimensional, Saturated-Unsaturated Groundwater Flow and Density dependent, Chain-decay Solute Transport in Porous, Discretely-Fractured Porous or Dual-Porosity Formations. User's guide (2003) 
Van Loon L.R., Soler, J.M, Jakob, A., Bradbury, M.H.: Effect of confining pressure on the diffusion of HTO, ${ }^{36} \mathrm{Cl}^{-}$and ${ }^{125} \mathrm{I}^{-}$in a layered argillaceous rock(Opalinus Clay): diffsion perpendicular to the fabric. Appl. Geochem 18, 1653-1662 (2003)

Van Marcke, Ph., Laenen, B.: The Ypresian Clays as Possible Host Rock for Radioactive Waste Disposal: An Evaluation, ONDRAF/NIRAS, Brussel (2005) 